EDITORIAL

\title{
ASPiH 2021 Conference - Moving \\ Upstream: Using simulation to improve systems
}

\author{
Michael Moneypenny ${ }^{1,2,3}$, Sharon Weldon ${ }^{4,5,6}$, Carrie Hamilton ${ }^{7}$, \\ Andy Buttery ${ }^{8,9}$, Guillaume Alinier $10,11,12,13$
}

\author{
${ }^{1}$ Clinical Skills, NHS Education, UK \\ ${ }^{2}$ Scottish Centre for Simulation and Clinical Human Factors, Larbert, UK \\ ${ }^{3}$ Forth Valley Royal Hospital, Larbert, UK \\ ${ }^{4}$ Institute of Lifecourse Development, University of Greenwich, London, UK \\ ${ }^{5}$ Barts Health NHS Trust, London, UK \\ ${ }^{6}$ Imperial College London, London, UK \\ ${ }^{7} \mathrm{Sim}$ Comm Academy, England, UK \\ ${ }^{8}$ Health Education England, Kent, Surrey \& Sussex, England \\ ${ }^{9}$ Institute of Medical Sciences, Faculty of Medicine, Health \& Social Care, Canterbury Christ \\ Church University \\ ${ }^{10}$ School of Health and Social Work, University of Hertfordshire, Hatfield, UK \\ ${ }^{11}$ Hamad Medical Corporation Ambulance Service, Doha, Qatar \\ ${ }^{12}$ Weill Cornell Medicine - Qatar, Doha, Qatar \\ ${ }^{13}$ Faculty of Health and Life Sciences, Northumbria University, Newcastle upon Tyne, UK
}

https://ijohs.com/article/doi/10.54531/WGMH9519

Consider these scenarios:

Simulation 1: A participant flushes a 1-year-old's cannula with $2 \%$ lidocaine instead of saline. Simulation 2 : An elderly patient is being prepared for discharge, in the absence of community support. Simulation 3: During a cardiac arrest, the charged defibrillator is powered off instead of being triggered to deliver a shock.

The debriefings are perfect. The participants discuss mental frames, the effects of stress, situation awareness, and many more ways that they could improve. But something is missing. There is no discussion about how the other parts of the system could be improved: Why look-alike medicines are still allowed, why roles and responsibilities are not always clearly defined, why equipment is not tested in the early design stages with real healthcare workers in realistic conditions.

In the modern era, simulation in healthcare was primarily used to 'improve' people, to 'make the person fit the task.' As our understanding of complex systems has evolved and the Safety-II paradigm has gained traction, the simulation community has started to appreciate the wider applicability of simulation to understand and improve the whole system

${ }^{[1]}$, to 'make the task fit the person'.
The concept of 'translational simulation' ${ }^{[2]}$ has the potential to make systemic improvements in healthcare which do not rely on the continuous education of healthcare professionals to make up for latent conditions. Additionally, patients are being included in simulation activities for purposes such as generating collaborative solutions to healthcare problems, and ensuring their voice is heard during the training of healthcare workers ${ }^{[3]}$. This adds a new dimension to the possibilities that simulation holds for the future of healthcare, moving it further away from the educational and 'clinical insider frame' that we are so accustomed to.

In last year's editorial, we celebrated the use of simulation in helping us adapt to the new ways of working imposed by COVID-19 [4]. In our quest to build on the lessons learnt, the ASPiH conference 2021 will connect enthusiasts in healthcare simulation, quality improvement, human factors, and patient involvement. The time has come to use our combined experience, expertise, and influence to move the focus from trying to 'improve' individuals to include the more impactful task of making healthcare systems safer for staff and patients ${ }^{[5]}$. They will thank us for it. 


\section{References}

1. Lamé G, Dixon-Woods M. Using clinical simulation to study how to improve quality and safety in healthcare. BMJ Simul Technol Enhanc Learn. 2020;6(2). Doi: 10.1136/ bmjstel-2018-000370

2. Nickson CP, Petrosoniak A, Barwick S, Brazil V. Translational simulation: from description to action. Adv Simul. 2021;6(1). Doi: 10.1186/s41077-021-00160-6
3. Kneebone R, Weldon SM, Bello F. Engaging patients and clinicians through simulation: rebalancing the dynamics of care. Adv Simul. 2016;15(1):19. Doi: 10.1186/s41077-016-0019-9

4. Moneypenny M, Alinier G, Attoe C, McCormack S. Simulation in the time of COVID 19. BMJ Simul Technol Enhanc Learn. 2020;6(Suppl 1):Ai-i.

5. Hignett S, Carayon P, Buckle P, Catchpole K. State of science: human factors and ergonomics in healthcare. Ergonomics. 2013;56(10):1491-1503. 